\title{
Begrüßung durch den Präsidenten des Verfassungsgerichtshofs
}

\author{
Spectabilis! \\ Liebe Kolleginnen und Kollegen! \\ Meine sehr geehrten Damen und Herren!
}

Gerne bin ich der Einladung gefolgt, im Rahmen der Eröffnung dieser heute beginnenden internationalen Tagung zum Thema "Normsetzung im Notstand - Außerordentliche Gesetzgebungsbefugnisse im 19. und 20. Jahrhundert" als Präsident des Verfassungsgerichtshofes Grußworte zu sprechen. Ich erachte diese Veranstaltung nämlich sowohl unter dem allgemein verfassungsrechtlichen Aspekt als auch ganz besonders im Hinblick auf die Verfassungsgerichtsbarkeit in Österreich als sehr verdienstvoll.

Mit dem Kriegswirtschaftlichen Ermächtigungsgesetz, ${ }^{1}$ dessen Erlassung sich als heuer zum 100. Mal jährt, wurde die Regierung des damals monarchischen Staates ermächtigt, "während der Dauer der durch den Krieg hervorgerufenen außerordentlichen Verhältnisse durch Verordnung die notwendigen Verfügungen zur Förderung und Wiederaufrichtung des wirtschaftlichen Lebens, zur Abwehr wirtschaftlicher Schädigungen und zur Versorgung der Bevölkerung mit Nahrungsmitteln und anderen Bedarfsgegenständen zu treffen"

(§ 1 Abs. 1).

Eine gleichartige Ermächtigung war bereits im Oktober 1914 durch Kaiserliche Verordnung i.S.d. § 14 des Grundgesetzes über die Reichsvertretung erteilt worden. ${ }^{2}$ Auf diese Kaiserliche Verordnung gründete praktisch das gesamte in

\footnotetext{
${ }^{1}$ Gesetz vom 24 . Juli 1917, mit welchem die Regierung ermächtigt wird, aus Anlass der durch den Kriegszustand verursachten außerordentlichen Verhältnisse die notwendigen Verfügungen auf wirtschaftlichem Gebiete zu treffen, RGBl. 307/1917.

${ }^{2}$ Kaiserliche Verordnung vom 10. Oktober 1914, mit welcher die Regierung ermächtigt wird, aus Anlaß der durch den Kriegszustand verursachten außerordentlichen Verhältnisse die notwendigen Verfügungen auf wirtschaftlichem Gebiete zu treffen, RGBl. 274/1914.
} 
den ersten Jahren des Ersten Weltkrieges aufgebaute kriegswirtschaftliche System. Dies allerdings ganz ohne Mitwirkung des Reichsrates, der zuletzt im März 1914 zusammengetreten war.

Vor diesem Hintergrund bedeutete das Kriegswirtschaftliche Ermächtigungsgesetz, das diese die Kaiserliche Verordnung ablöste, insoferne einen gewissen rechtsstaatlichen Fortschritt, als es darauf abzielte, die staatliche Kriegswirtschaft auch für die Vergangenheit auf eine vom Reichsrat sanktionierte verfassungsmäßige Grundlage zu stellen. ${ }^{3}$

Die Erste Republik hielt am Kriegswirtschaftlichen Ermächtigungsgesetz ausdrücklich fest. § 7 Abs. 2 ÜbergangsG 1920 ordnete an, dass die nach diesem Gesetz der Regierung zustehenden Befugnisse sowohl auf die Bundesregierung als auch auf die einzelnen Bundesminister übergehen. Mit dieser Bestimmung war - so Hans Kelsen - „das bisher verfassungsrechtlich bedenkliche kriegswirtschaftliche Ermächtigungsgesetz vom Standpunkt der neuen Bundesverfassung aus legitimiert".

Der Zeitpunkt, von dem an die durch den Weltkrieg verursachten ",außerordentlichen Verhältnisse" als behoben anzusehen sind, war durch Bundesgesetz festzustellen (§ 17 Abs. 2 ÜbergangsG 1920). Ein entsprechendes Bundesgesetz wurde aber - wiederholten Mahnungen zum Trotz - nicht erlassen.

Und so wurde es möglich, dass nach der Ausschaltung des Nationalrates am 4. März 1933 dieses Kriegswirtschaftliche Ermächtigungsgesetz dann jenen Kräften in die Hände spielte, die sich zum Ziel gesetzt hatten, Österreich nach dem Beispiel des Deutschen Reiches einem autoritären Regime zu unterwerfen: Gestützt auf das Kriegswirtschaftliche Ermächtigungsgesetz, erließ die Bundesregierung in der Folge eine ganze Reihe "kriegswirtschaftlicher" Verordnungen; so auch mehrere, mit denen verfassungsgesetzlich gewährleistete Rechte, also Grundrechte, gezielt beschränkt wurden. Hervorzuheben sind etwa die gegen die Pressefreiheit gerichtete Verordnung vom 7. März 1933 betreffend besondere Maßnahmen zur Hintanhaltung der mit einer Störung der öffentlichen Ruhe, Ordnung und Sicherheit verbundenen Schädigungen des wirtschaftlichen Lebens, die Plakatierungsverordnung vom 26. April 1933 oder die Verordnung vom 19. Mai 1933 zur Hintanhaltung politischer Demonstrationen. Dass diese Verordnungen früher oder später beim Verfassungsgerichtshof angefochten werden - und der Aufhebung verfallen - würden, galt als höchst wahrscheinlich.

Um einer für das Regime ungünstigen Entscheidung des Verfassungsgerichtshofes zuvorzukommen, wurde sodann am 23. Mai 1933 eine weitere „kriegswirtschaftliche" Verordnung erlassen, die es den vom Nationalrat und vom

\footnotetext{
${ }^{3}$ Bericht des kriegswirtschaftlichen Ausschusses des Abgeordnetenhauses, 429 BlgAH 22. Session 2.

${ }^{4}$ Hans Kelsen, Georg Froehlich, Adolf MerkL, Die Bundesverfassung vom 1. Oktober 1920 (= Verfassungsgesetze der Republik Österreich 5, Wien 1922) 311.
} 
Bundesrat nominierten Mitgliedern und Ersatzmitgliedern des Verfassungsgerichtshofes untersagte, an den Beratungen des Gerichtshofes teilzunehmen, „wenn und solange dem Verfassungsgerichtshof [nicht] sämtliche Mitglieder und Ersatzmitglieder angehören, die auf Grund solcher Vorschläge ernannt worden sind". ${ }^{5}$ Im Hinblick auf diese Regelung traten in der Folge - in einer konzertierten Aktion - sieben auf diese Weise ernannte, der Bundesregierung nahestehende Mitglieder bzw. Ersatzmitglieder zurück, sodass der Gerichtshof nicht mehr das für eine Beschlussfassung im Plenum erforderliche Anwesenheitsquorum (damals wie heute: Vorsitzender und acht Stimmführer) erreichte. Der Verfassungsgerichtshof war damit "lahmgelegt" - und mit diesem "Schachzug" gleichzeitig die letzte juristische Hürde auf dem Weg Österreichs in die Diktatur aus dem Weg geräumt.

Die Art und Weise, in der dies erfolgte, hat Robert Walter in seiner Festrede anlässlich des Verfassungstages 1997 sehr treffend wie folgt beschrieben: „[Die ,Ausschaltung' des Verfassungsgerichtshofes] wurde durch eine - juristisch kunstvoll ersonnene - Verordnung, aus deren Schlinge sich der VfGH nicht zu befreien vermochte, herbeigeführt". Als spiritus rector dieser Aktion fungierte bekanntlich Sektionschef Robert Hecht, dessen für Rechtsstaat und Demokratie zerstörerisches Wirken in einem äußerst lesenswerten Buch von Peter Huemer sehr anschaulich beschrieben wurde. ${ }^{6}$

Über diese für die Geschichte der österreichischen Verfassungsgerichtsbarkeit so bedeutsamen Ereignisse hier vor Ihnen kurz zu sprechen, ist mir ein besonderes, ein persönliches Anliegen: und zwar deshalb, weil das, was damals im Jahr 1933 geschehen ist, immer wieder geschehen kann. Die Art und Weise etwa, wie die in Polen seit der letzten Parlamentswahl regierende Mehrheit das dortige Verfassungstribunal zunächst weitgehend lahmgelegt und in der Folge offenbar gezielt mit eigenen Parteigängern besetzt hat, ähnelt in mancher Hinsicht frappierend jenen Methoden, die das seinerzeitige austrofaschistische Regime bei der Ausschaltung des österreichischen Verfassungsgerichtshofes angewendet hatte. Die geschilderte Entwicklung in Polen ist übrigens deshalb besonders schmerzlich, weil sich das polnische Verfassungstribunal in den Jahrzehnten nach der politischen Wende in Osteuropa unter den europäischen Verfassungsgerichten einen ausgezeichneten Ruf erworben und eine ganz bemerkenswerte Vorbildwirkung für andere Verfassungsgerichte ausgeübt hat. Zudem birgt das Vorgehen der polnischen Regierung und der dortigen Parlamentsmehrheit die Gefahr einer negativen Beispielswirkung für andere Länder der Region oder in Europa insgesamt in sich!

\footnotetext{
${ }^{5} \S 6$ Abs. 3 VfGG i.d.F. der Verordnung der Bundesregierung vom 23. 5. 1933, betreffend Abänderungen des Verfassungsgerichtshofgesetzes 1930, BGBl. 191/1933.

${ }^{6}$ Peter HuEMER, Sektionschef Robert Hecht und die Zerstörung der Demokratie in Österreich. Eine historisch-politische Studie (Wien 1975).
} 
Ein Blick auf das Programm dieser Veranstaltung zeigt mir, dass über das Kriegswirtschaftliche Ermächtigungsgesetz als Wegbereiter des autoritären Ständestaates oder das Verhältnis von Verfassungsgerichtsbarkeit und Notverordnung im Rahmen dieser Veranstaltung ohnedies - von Berufeneren, als ich es dafür bin, - detailliert referiert werden wird. Mir ist es aber als Präsident des Verfassungsgerichtshofes ein ganz besonderes Anliegen, darauf hinzuweisen, dass so manches, was Sie im Rahmen dieser Veranstaltung als historischen Vorgang behandeln werden, durchaus aktuelle Bedeutung hat. Insoferne liegt der Wert dieser Tagung im Besonderen auch darin, nachhaltig dafür zu sensibilisieren, dass Rechtsstaat und Demokratie auch in unserer Zeit keine "Selbstläufer", keine Selbstverständlichkeit sind, sondern überall - auch bei uns in Österreich! - tagtäglich aufs Neue in ihrem Bestand bewahrt und fortentwickelt werden müssen!

Wien, 16. Oktober 2017

Dr. Gerhart HolzingeR 